\title{
Perfil cronobiológico, aptidão física e sonolência diurna excessiva em escolares
}

Compreender a Cronobiologia é saber como acontece o processamento do ritmo biológico do organismo do ser humano. A aptidão física é principalmente estimulada durante a infância e juventude, pois é nessa fase onde os sistemas motor e cognitivo estão mais vulneráveis a aprendizagem. Justifica-se a escolha do tema por sentir necessidade de aprofundar o conhecimento teórico e prático acerca do assunto. $O$ objetivo dessa pesquisa é analisar o perfil cronobiológico, aptidão física e a sonolência excessiva diurna em escolares de ambos os sexos do município de Lavras da Mangabeira/CE. A seguinte pesquisa se caracteriza de campo, intencional, composta por 72 alunos, de escolas da rede pública de ensino médio. Os dados foram coletados através do questionário de Horne e Ostberg, tabela de sonolência e testes do Proesp Brasil, a análise dos dados foi realizada por meio de estatística descritiva. Os resultados nos mostram que em sua maioria os alunos estão dentro da zona saudável, possuem perfil cronobiológico vespertino, em apenas um teste o resultado encontrado foi negativo. Com tudo se conclu que devemos nos atentar ainda mais para informações e procurar ainda mais conhecimento para que possamos intervir de maneira eficaz, cessando problemas existente.

Palavras-chave: Cronobiologia; Aptidão física; Sonolência diurna.

\section{Chronobiological profile, physical fitness and excessive daytime sleepiness in schoolchildren}

\begin{abstract}
Understanding Chronobiology is knowing how the biological rhythm of the human organism is processed. Physical fitness is mainly stimulated during childhood and youth, as it is at this stage where the motor and cognitive systems are most vulnerable to learning. The choice of the theme is justified because it feels the need to deepen the theoretical and practical knowledge about the subject. The objective of this research is to analyze the chronobiological profile, physical fitness and excessive daytime sleepiness in schoolchildren of both sexes in the municipality of Lavras da Mangabeira/CE. The following research is characterized by field, intentional, composed of 72 students, from public high schools. Data were collected using the Horne and Ostberg questionnaire, sleepiness table and Proesp Brasil tests. Data analysis was performed using descriptive statistics. The results show us that the majority of students are within the healthy zone, have an evening chronobiological profile, in only one test the result found was negative. However, it is concluded that we must pay even more attention to information and seek even more knowledge so that we can intervene effectively, ceasing existing problems.
\end{abstract}

Keywords: Chronobiology; Physical aptitude; Daytime sleepiness.

Topic: Educação Física

Reviewed anonymously in the process of blind peer.
Received: 10/02/2020

Approved: 19/05/2020
Cícero Cleber Brito Pereira (DD)

Centro Universitário Vale do Salgado, Brasil http://lattes.cnpq.br/6554764959422181 http://orcid.org/0000-0002-9402-9810

cicerocleber@univs.edu.br

Carolina Landim Gomes (it)

Centro Universitário Vale do Salgado, Brasil

http://lattes.cnpq.br/8133512876478249

http://orcid.org/0000-0001-5033-4891

carolmorais1@outlook.com

Gabriela Gomes de Oliveira Bezerra (iD

Centro Universitário Dr. Leão Sampaio, Brasil

http://lattes.cnpq.br/6330341118187369

http://orcid.org/0000-0002-9724-708X

gabriela.o.bezerra@gmail.com
Marcos Antônio Araújo Bezerra (iD)

Centro Universitário Dr. Leão Sampaio, Brasil

http://lattes.cnpq.br/4643352879633283

http://orcid.org/0000-0002-3385-4024

marcosantonio@leaosampaio.edu.br
Referencing this:

PEREIRA, C. C. B.; GOMES, C. L.; BEZERRA, G. G. O.; BEZERRA, M. A. A.. Perfil cronobiológico, aptidão física e sonolência diurna excessiva em escolares. Medicus, v.2, n.2, p.18-24, 2020. DOI: http://doi.org/10.6008/CBPC2674-6484.2020.002.0003 


\section{INTRODUÇÃO}

São considerados fatores importantes que influem no processo de sono-vigília nos humanos alguns deles são: sexo, idade, perfil cronobiológico, duração do sono e variações genéticas. Desde os primórdios o ciclo claro/escuro é avaliado o mais relevante dos ritmos de mamíferos, já que eles se guiavam pela luz do sol, lua e estrelas, porém nos seres humanos, depois da invenção da energia elétrica, os parâmetros de sincronização foram mudados já que agora há luz mesmo a noite, nos casos de situações de trabalho e estudo noturno, viagens e costumes agora adotados como televisão e internet, estão associados à dessincronizarão dos ritmos circadianos. Com isso podem acorrer dificuldades para adormecer e acordar, isso pode refletir diretamente no trabalho ou estudo, ao tentar cumprir com todos os compromissos, o ser pode ocasionar uma perca de horas de sono podendo adquirir sonolência diurna excessiva (SANTOS et al., 2016).

Segundo Pereira et al. (2016), os jovens no Brasil, não atingem os níveis ideais de aptidão física, ele fala ainda que problemas relacionados a saúde dos mesmos estão relacionados ao baixo nível de aptidão física, lembrando que aptidão física é estimulada na infância. No entanto ainda hoje por muitas vezes sabemos que na escola, não consta um professor formado em educação física que possui embasamento teórico e prático para que a criança seja estimulada da maneira correta, e com isso aumentando seu acervo motor tendo melhora na coordenação motora, resistência física e melhor no cognitivo.

A redução do sono e a dificuldade de se manter ativo durante o dia, acontece devido a perturbação do ciclo de sono-vigília podendo afetar o desempenho físico e cognitivo, favorecendo o desenvolvimento de doenças cardiovasculares e metabólicas, elas podem ser diabetes e obesidade, por isso o distúrbio do ciclo sono-vigília tem sido considerado um problema de saúde pública que precisa de medidas e estratégias para seu tratamento, é ai onde entra a atividade física que é uma importante aliada, trazendo melhora na saúde física e social, aumentando a capacidade funcional e qualidade de vida (BERNARDO et al., 2018).

Em relação ao sono entende-se que este é essencial para o crescimento físico, a estabilidade emocional, o comportamento e a manutenção da função cognitiva do indivíduo, em especial do adolescente, justificando que este se encontra nesse período em crescimento e precisa preservar seu sono em total equilíbrio de tempo, para não perder o potencial de sua memória, o que teria sérias consequências e implicações para o seu sucesso na escola. Uma noite de sono com qualidade refletirá no melhor desempenho escolar e na maior motivação para os estudos. Os pesquisadores no assunto garantem que a população de adolescentes precisa de em média oito a nove horas de sono toda noite para desempenhar de forma adequada suas atividades cotidianas, bem como absorvem os benefícios físicos e mentais que o sono proporciona (VILELA et al., 2016).

O estudo desenvolvido tem sua justificativa a partir da necessidade de conhecer sobre as teorias relacionadas ao tema, percebendo o quão é importante investigar sobre o perfil cronobiológicos, aptidão física e a sonolência excessiva diurna, haja vista a pretensão de buscar respostas para o problema existente. Segundo Amaro et al. (2018), a falta do sono também pode ser prejudicial ao desempenho cognitivo que envolve o aprendizado, o raciocínio lógico, a memória, os cálculos matemáticos, processamento verbais 
complexos, e tomadas de decisões. Porem na sociedade moderna há a necessidade de trabalhar em horários irregulares, compromissos sociais durante a noite, o que pode modificar os mecanismos da qualidade do sono, sendo capaz de ocorrer estragos à saúde física e mental do ser humano. Este estudo tem como finalidade de identificar o perfil cronobiológico, nível de aptidão física e a sonolência diurna excessiva em escolares em turmas do 3o ano do ensino médio no município de Lavras da Mangabeira/CE. Sendo aplicados testes e questionários.

\section{METODOLOGIA}

\section{Caracterização e Participantes}

A seguinte pesquisa se caracteriza de campo, intencional, composta por 72 alunos de ambos os sexos de escolas da rede pública de ensino médio, as escolas visitadas foram: Escola de Ensino Médio Filgueiras Lima (35); Escola em Tempo Integral Alda Ferrer Augusto Dutra (19) e Escola Profissional Professor Gustavo Augusto Lima (18) no município de Lavras da Mangabeira/CE.

Os alunos que tiveram sua participação tinham faixa etária entre 15 a 17 anos. 0 tamanho da amostra foi de 72 alunos que estavam dentro dos critérios de inclusão e exclusão, que são: ser aluno de uma das instituições de ensino citadas acima, estar estudando o 3 o ano do ensino médio, situar-se devidamente matriculado e frequentando as aulas, estar na faixa etária, aceitar participar de todas as fases da bateria de testes e aceitar responder ao questionário condicionada a entrega do TCLE. Os critérios de exclusão foram: Estar ausente em algumas das etapas da pesquisa, não está com trajes adequados para a realização dos testes, possuir algum tipo de limitação física devidamente comprovada que o impeça de realizar alguma das fases do teste, não aceitar responder os questionários.

\section{Instrumentos e Procedimentos}

A pesquisa será efetivada mediante a aplicação de dois questionários, o questionário de perfil cronobiológico aplicado será o de Horne e Ostberg, que possui escala de 70 a 86 - matutino típico, 59 a 69 moderadamente matutino; 42 a 58 -indiferente, 31 a 41 -moderadamente vespertino, 16 a 30 -vespertino típico. O segundo questionário será a escala de Epworth, que tem como escala 0 a 10 normal; 11 a 16 sonolência leve; 17 a 20 sonolência moderada; 21 a 24 sonolência severa.

Os dois questionários terão seus resultados a partir da soma de seus escores, contara também com aplicação de teste do PROESP BRASIL relacionados a aptidão fisica, os testes foram IMC que se utilizou uma balança digital da marca supermedy, foi também utilizado uma trena de fibra da marca sanny fixada na parede um metro acima do solo, perímetro da Cintura (CC) a medida foi verificada por meio de uma fita métrica, do ponto central entre a borda inferior da última costela e crista ilíaca.

O próximo teste é a, razão cintura estatura (RCE) que é definido por meio do cálculo de divisão entre a medida da circunferência da cintura em centímetros e também da estatura (altura) em centímetros, teste da aptidão cardiorrespiratória (corrida/caminhada dos 6 minutos), prevenindo corridas de velocidade 
mescladas com longas caminhadas, teste de flexibilidade (teste de sentar e alcançar) com uma fita estendida no solo, a fita adesiva deverá ser fixada no solo, o aluno terá que estar sem tênis, com os calcanhares em cima da fita adesiva marcando 38 centímetros e os joelhos separados a 30 centímetros, com os joelhos estendidos e as mãos uma acima da outra, sendo realizadas duas tentativas.

Todos os participantes foram informados dos procedimentos a serem adotados na pesquisa. Após aprovação e aceite da metodologia a ser empregada, os participantes foram orientados a assinar um Termo de Consentimento Livre e Esclarecido (TCLE) em acordo a resolução 466/12 do Conselho Nacional de Saúde. A análise se deu a partir dos dados coletados dos questionários e testes, com base na soma dos escores coletados, foi observado a classificação na qual o aluno se encaixava e com isso pode-se identificar os níveis de sonolência e seu perfil cronobiológico, após a aplicação dos questionários os alunos foram submetidos aos testes, nos quais foram obtidos números e através dos mesmos classificou-se os níveis de aptidão física. O projeto foi submetido e aprovado ao Comitê de Ética em Pesquisa do Centro Universitário Dr. Leão Sampaio (UNILEÃO) como número de parecer 3.704.572.

\section{Análise de Dados}

Foi utilizado o software JASP na versão 0.9.0.1, para análise dos dados através de estatística descritiva por distribuição de frequência.

\section{RESULTADOS E DISCUSSÃO}

A amostra se deu com 72 discentes de ambos os sexos do terceiro ano do ensino médio das escolas públicas de Lavras da Mangabeira, com as medias de Idade $16,90 \pm 0,30$ para o sexo masculino e $16,87 \pm 0,33$ para feminino, com massa corporal $61,90 \pm 8,37$ no sexo masculino e $53,11 \pm 7,81$ para sexo feminino,

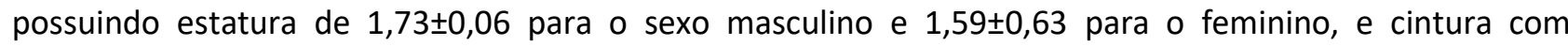
$71,45 \pm 4,23$ para o sexo masculino e $66,02 \pm 7,09$ para o feminino.

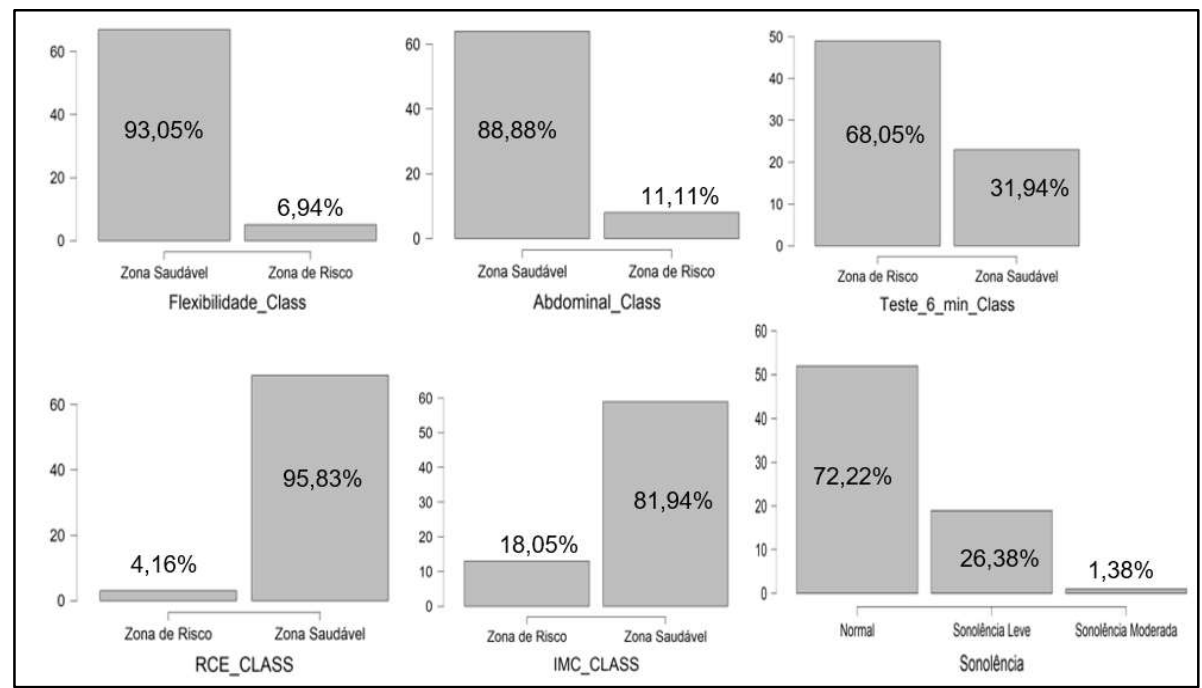

Gráfico 1: Valores de classificação da flexibilidade, resistência abdominal, capacidade cardiorrespiratória, relação cintura estatura, índice massa corporal e níveis de sonolência.

Os resultados do presente estudo nos deram os seguintes dados sobre sonolência, a maioria dos 
alunos tem os níveis de sonolência considerado normais, alguns têm sonolência leve e apenas uma minoria sonolência moderada. De maneira geral, há algum tempo a privação de sono é o fator principal associado à sonolência excessiva, além disso, outros fatores biológicos, comportamentais e ambientais podem contribuir para uma diminuição das horas de sono, como para o aumento da sonolência diurna excessiva (ROCHA et al., 2009).

Os dados relacionados aos testes do PROESP BRASIL de Flexibilidade nos mostram que a maior parte da população de alunos está na zona saudável e uma pequena porcentagem na zona de risco. Encontrou-se no teste de 6 minutos, onde os maiores números dos alunos estão dentro da zona de risco e apenas alguns por cento estão na zona saudável. Já no teste de RCE (relação cintura estatura), na qual quase todos estão dentro da zona saudável e somente alguns indivíduos dentro da zona de risco. Nos valores de IMC (índice de massa corporal), onde na sua maioria estão dentro da zona saudável e só alguns na zona de risco, já no teste de resistência abdominal, percebemos que em sua maioria os alunos estão dentro da zona saudável onde apenas uma pequena porcentagem se encontra em zona de risco.

$\mathrm{Na}$ aplicação dos questionários, verificou-se que todos os alunos possuem perfil cronobiológico Vespertino Típico, o critério usado para comparação foi o do questionário de Horne e Ostberg (inserir ano), 70 a 86 - matutino típico; 59 a 69 - moderadamente matutino; 42 a 58 - indiferente; 31 a 41 moderadamente vespertino; 16 a 30 - vespertino típico. A pessoa é caracterizada como vespertino porque se adapta melhor a este período que compreende o horário das doze horas em diante. Assim, o perfil vespertino tem relação direta com o final da tarde e início da noite, pois, entende-se que é quando os indivíduos se sentem mais indispostos para realizar as suas atividades cognitivas, diária (ALAM, 2012).

Para os resultados da escala de sonolência, foi utilizado a escala de Epworth, que tem como escores 0 a 10 normal; 11 a 16 sonolência leve; 17 a 20 sonolência moderada; 21 a 24 sonolência severa. Para os resultados dos testes do PROESP, foi levado em conta as medidas de segurança de zona saudável, oferecido pelo próprio site.

Tabela 1: Resultados do PROESP por sexo.

\begin{tabular}{lllll}
\multirow{2}{*}{ AFRS } & \multicolumn{3}{l}{ Descritiva } & Mulheres \\
\cline { 2 - 5 } & Homens & & Média & DP \\
\cline { 2 - 5 } & Média & DP & 20,88 & 2,65 \\
IMC & 20,79 & 3,19 & 0,41 & 0,36 \\
RCE & 0,41 & 0,03 & 39,14 & 9,51 \\
FLEX & 40,35 & 8,03 & 15,31 & 3,85 \\
N $^{\circ}$ ABD & 27,71 & 7,73 & 886,85 & 94,19 \\
APT CARD & 1492,58 & 361,71 & & \\
\hline
\end{tabular}

Durante o estudo, podemos observar que somente no teste de resistência de 6 minutos de corrida, os alunos entraram em sua maioria na zona risco, tento os escores de zona de corte sendo 1,119 m para o sexo masculino e 1,079 m, para o sexo feminino, com base nos números encontrados, observamos que as meninas tem a taxa de corte menor que os meninos, o que também foi encontrado no estudo de Minatto et al. (2015) que mostra o baixo percentual de aptidão cardiorrespiratória, em alunos de Januária/MG.

Também no estudo de Silva et al. (2010), que mostra os resultados semelhantes e Silva et al. (2015), também afirmou ter encontrado mais meninos com baixos índices de aptidão física em relação as meninas. 
Ferrari et al. (2015), mostraram que durante os 30 anos de estudos aparentemente aconteceram uma baixa nos níveis de aptidão física dos escolares de llhabela. Lembrando que os resultados dos estudos se deram por métodos diferentes.

Estudos realizados pelo PROESP BRASIL revelam que certos níveis de aptidão cardiorrespiratória (teste dos 6 minutos) podem ter haver com a ocorrência de níveis elevados de colesterol, hipertensão arterial e resistência à insulina. Nos demais testes contatou-se que níveis estão dentro da zona de saúde, comparados com os parâmetros oferecidos pelo mesmo.

Coledam et al. (2016) nos diz que existe uma associação entre um avanço da aptidão cardiorrespiratória, com alunos praticantes das aulas de educação física, as quais normalmente são aplicadas duas aulas por semana em cada turma, com isso os alunos são estimulados a se manterem sempre ativos, fazendo algum tipo de atividades físicas, sendo elas brincadeiras ou algum tipo de esporte.

O referente estudo vem nos mostrar que a população masculina 40,35\% teve sua flexibilidade maior que população feminina com 39,40\%, no estudo de Pereira et al. (2016) encontramos resultados contrários, mostrando que o público feminino possui mais flexibilidade que o masculino. Os trabalhos tiveram os mesmos testes para aferir os resultados. Segundo Martins-costa et al. (2015) outros fatores como a sequência do crescimento dos segmentos corporais, podem interferir no desempenho normal, identificado nos anos da puberdade pelo teste de flexibilidade de sentar e calçar.

Já Godoi Filho et al. (2015) nos fala que as variáveis relacionadas à aptidão física durante a puberdade apontaram resultados crescentes com o passar da idade, porem em relação a flexibilidade observou-se que durante os 12 e 13 anos de idade aconteceu um declínio, onde veio a melhorar na chegada dos 18 anos, os resultados baixos encontrados na puberdade, podem estar relacionados ao crescimento nos membros inferiores e tronco-cefálico.

\section{CONCLUSÕES}

O presente estudo constatou que os alunos do terceiro ano das escolas públicas de Lavras da Mangabeira, possuem perfil cronobiológico vespertino típico, estão dentro da zona saudável nos parâmetros, flexibilidade, abdominais, sonolência, relação cintura estatura, mas no teste de aptidão cardiorrespiratória (teste de 6 minutos) os alunos em sua maioria entrarão em zona de risco.

Isso nos mostra o quão importante é conhecer a realidades dos discentes, e que temos, que pesquisar mais sobre o tema para que possamos entender os fatores externo que influenciam no desempenho dos alunos. Para que possamos criar novas estratégias de ensino aprendizagem com base no estilo de vida levado pelas novas gerações. Investir nesse campo de pesquisa nos possibilitara entender e compreender os fatores intrínsecos e extrínsecos existentes, que influenciam nos resultados finais dos alunos, com isso existirá um meio termo entre escola e estudante, podendo existir assim uma melhora no desempenho final do aluno.

\section{REFERÊNCIAS}

ALAM, M. F.. A relevância da cronobiologia no processo

saúde-doença: cronotipo com o estilo de vida e saúde. Porto

Alegre: UFRGS, 2012 
AMARO, J. M. R. S.; DUMITH, S. C.. Sonolência diurna excessiva e qualidade de vida relacionada à saúde dos professores universitários. Jornal Brasileiro de Psiquiatria, Rio de Janeiro, v.67, n.2, p.94-100, 2018.

BERNARDO, V. M.; SILVA, F. C.; FERREIRA, E. G.; BENTO, G. G.; SILCH, M. C.; SOUSA, B. A.; SILVA, R.. Atividade física e qualidade de sono em policiais militares. Revista Brasileira de Ciências do Esporte, Brasília, v.40, n.2, p.131-137, 2018. DOI: https://doi.org/10.1016/j.rbce.2018.01.011

COLEDAM, D. H. C.; FERRAIOL, P. F.; SANTOS, J. W.; OLIVEIRA, A R.. Fatores associados à aptidão cardiorrespiratória de escolares. Revista Brasileira de Medicina do Esporte, São Paulo, v.22, n.1, p.21-26, 2016

FERRARI, G. L. M.; MATSUDO, V. K. R.; FISBERG, M.. Modificações da aptidão física e estado nutricional de escolares em um período de 30 anos (1980-2010). Revista Paulista de Pediatria, São Paulo, v.33, n.4, p.415-422, 2015.

GODOI FILHO, J. R. M.; FARIAS, E. S.. Aptidão física de escolares do sudoeste da Amazônia Ocidental em diferentes estágios de maturação sexual. Revista Brasileira de Educação Física e Esporte, São Paulo, v.29, n.4, p.631-639, dez. 2015.

HORNE J. A.; OSTGBERG, O.. A self-assessment questionnaire to determine morningness-eveningness in human circadian rhythms. Int. J. Chronobiology, v.4, p.97-110, 1976.

MARTINS-COSTA, H. C.; ARAUJO, S. R. S.; LIMA, F. V.; MENZEL, H.-J.. Análise do perfil da flexibilidade de crianças e adolescentes mensurada por meio de dois testes. Revista da Educação Física, Maringá, v.26, n.2, p.257-257, 2015. DOI: https://doi.org/10.4025/reveducfis.v26i2.22871
MINATTO, G.; SILVA, D. A. S.; PELEGRINI, A. FIDELIX, Y. L.; SILVA, A. F.; PETROSKI, E. L.. Aptidão cardiorrespiratória, indicadores sociodemográficos e estado nutricional em adolescentes. Revista Brasileira de Medicina do Esporte, São Paulo, v.21, n.1, p.12-16, 2015.

PEREIRA, T. A.; BERGMANN, M. L A.; BERGMANN, G. G.. Fatores associados à baixa aptidão física de adolescentes. Revista Brasileira de Medicina do Esporte, São Paulo, v.22, n.3, p.176-181, 2016.

ROCHA, M. C. P.; MARTINO, M. M. F.. Estresse e qualidade do sono entre enfermeiros que utilizam medicamentos para dormir. Acta Paulista de Enfermagem, São Paulo, v.22, n.5, p.658-665, 2009.

SANTOS, T. C. M. M.; MARTINO, M. M. F.; SONATI, J. G.; FARIA, A. L.; NASCIMENTO, E. F. A.. Qualidade do sono e cronotipo de estudantes de enfermagem. Acta Paulista de Enfermagem, São Pulo, v.29, n.6, p.658-663, 2016. DOI: https://doi.org/10.1590/1982-0194201600092

SILVA, S. P.; SANTOS, A. C. S.; SILVA, H. M.; COSTA, C. L. A.; NOBRE, G. C.. Aptidão cardiorrespiratória e composição corporal em crianças e adolescentes. Motriz Revista de Educação Física, Bela Vista, v.16, n.3, p.7-8, 2010.

SILVA, D. A. S.; TREMBLAY, M. S.; PELEGRINI, A.; SILVA, J. M. F. L.. Low aerobic fitness in Brazilian adolescents. Revista Brasileira de Medicina do Esporte, São Paulo, v.21, n.2, p.94-98, 2015. DOI: https://doi.org/10.1590/1517$\underline{869220152102144547}$

VILELA, T. S.; BITTENCOURT, S. T.; MOREIRA, G. A.. Fatores que influenciam na sonolência excessiva diurna em adolescentes. Jornal da Pediatria, Porto Alegre, v.92, n.2, p.149-155, 2016

A CBPC - Companhia Brasileira de Produção Científica (CNPJ: 11.221.422/0001-03) detém os direitos materiais desta publicação. Os direitos referem-se à publicação do trabalho em qualquer parte do mundo, incluindo os direitos às renovações, expansões e disseminações da contribuição, bem como outros direitos subsidiários. Todos os trabalhos publicados eletronicamente poderão posteriormente ser publicados em coletâneas impressas sob coordenação da Cognitionis Publishing, da Companhia Brasileira de Produção Científica e seus parceiros autorizados. Os (as) autores (as) preservam os direitos autorais, mas não têm permissão para a publicação da contribuição em outro meio, impresso ou digital, em português ou em tradução. 